\title{
The secretome of Serratia marcescens SM6 under oxidative stress conditions
}

\author{
L.E. Matrosova ${ }^{1 *}$, I.V. Khilyas ${ }^{1}$, L.M. Bogomolnaya ${ }^{1,2}$ \\ ${ }^{1}$ Institute of Fundamental Medicine and Biology KFU, Kazan, Russia \\ ${ }^{2}$ Texas A\&M University Health Science Center, Bryan, Texas, USA \\ *e-mail: M.Lilia.Evg@yandex.ru
}

Key words: efflux system, MacAB, secretome, Serratia

Motivation and Aim: Multidrug efflux pumps representing the membrane protein complexes play a significant role in a drug resistance and natural physiology of pathogenic bacteria such as $S$. marcescens [1]. Four different classes of efflux pumps were identified in the genome of $S$. marcescens. Clear understanding of the natural functions of efflux pumps is required for the development of new generation of antimicrobials to combat bacterial drug resistance. The objective of this work was to study the antioxidant effect of extracellular metabolites produced by environmental strain S. marcescens SM6 in response of oxidative stress and secreted in efflux pump MacAB-dependent fashion.

Methods and Algorithms: All experiments were performed in $\mathrm{M} 9$ medium at $37^{\circ} \mathrm{C}$ in the presence/absence of hydrogen peroxide. Cell-free growth media used for cultivation of $S$. marcescens wild type SM6 or $\triangle m a c A B$ strains were passed through Supelco Discovery DSC-18 solid phase extraction C18 cartridges. Purification and fraction collection of metabolites was performed on an Acclaim ${ }^{\circledR}$ PolarAdvantage II (PA2) C18 reverse-phase column using UltiMate 3000 UHPLC system (Thermo Scientific, Dionex, USA).

Results: Cultivation of $\triangle m a c A B S$. marcescens mutant strain in the presence of a hydrogen peroxide led to a drastic drop in bacterial viability. Interestingly, extracellular metabolites produced by $S$. marcescens SM6 wild type under oxidative stress conditions had a protective effect on the growth of $S$. marcescens $\triangle m a c A B$ in the presence of peroxide. HPLC analysis detected several unique peaks with retention time of 12.360 , $12.757,19.237,23.317$ и $24.797 \mathrm{~min}$, respectively, that were present only in the extracts from conditioned media used for growth of $S$. marcescens wild type but not the $\triangle m a c A B$ mutant strain. Addition of these fractions to the peroxide-containing media rescued growth of $\triangle m a c A B$ mutant strain.

Conclusion: Results of this study show that hydrogen peroxide affected the composition of secreted metabolites of S.marcescens SM6. Particularly, HPLC analysis determined the presence of several peaks in the samples of the conditioned media used for growth of wild type strain SM6 under stress conditions.

Acknowledgements: This work was supported by the Russian Science Foundation project 16-14-10200 and performed in accordance with the Russian Government Program of Competitive Growth of Kazan Federal University.

\section{References}

1. Mahlen S.D. (2011) Serratia infections: from military experiments to current practice. Clin Microbiol Rev. 24(4):755-791.

2. Nishino K. (2005) Bacterial multidrug exporters: insights into acquisition of multidrug resistance. Science [online publication]. 2005. http://www.sciencemag.org/fea\$ture/data/prizes/ge/2004/nishino.dlt 